\title{
Singularities Analysis of the Jacobian Matrix Modified in the Continuation Power Flow: Mathematical Modeling
}

\author{
A. Bonini Neto and D. A. Alves
}

\begin{abstract}
In recent years, the concern for voltage stability issue has gained global highlighted when referring to the energy sector industry, this is because this issue is related to the operation and planning of electrical power systems. Factors such as the increasing energy demand, the transfer of large amounts of power to meet the consumption, combined with the economic and environmental requirements has led the systems operate in stressful conditions. In the literature, a study to analyze stability and voltage instability is related to the $P-V$ curve (power versus voltage magnitude) and the maximum loading point (MLP) (point on the curve that separates the stable operation of the unstable). The maximum loading point may be consequent to a saddle node bifurcation (SNB) related to transmission capacity limit in an electrical system where the Jacobian matrix is singular, or limit induced bifurcation (LIB), related the reactive power limit of the generator, where the matrix is not singular. In this sense, it is presented in this first part of the paper, a flowchart for the determination of the Jacobian matrix determinants modified for large systems such as two realistic systems of 638 and 787-bus corresponding to part of SouthSoutheast Brazilian system and of a 904-bus Southwestern American system. Also presented an analysis of the modified Jacobian matrices $\left(J_{m}\right)$ of the methods of continuation power flow (CPF) reported in the literature (parameterization methods), the study was developed in order to analyze the changes that the matrix conventional Jacobian (J) have to eliminate the singularity problems in the MLP and in the bifurcation points of each method.
\end{abstract}

Keywords - Voltage stability, Maximum loading point, Bifurcation, Continuation power flow, Continuation method.

\section{INTRODUÇÃO}

A TUALMENTE com o aumento crescente da demanda de energia elétrica, os estudos dos métodos de fluxo de carga continuado têm aumentado muito ao redor do mundo. A importância destes métodos se deve ao fato deles possibilitarem a obtenção do $\mathrm{PMC}$ com uma precisão préestabelecida e sem problemas numéricos, o traçado completo das curvas $\mathrm{P}-\mathrm{V}$ e a determinação da margem segura de carregamento. Vários métodos foram desenvolvidos visando obter toda a curva P-V e eliminar os problemas numéricos relacionados à matriz $\mathbf{J}$ [1-6]. Em [1] foi proposto um método da continuação utilizando uma técnica de parametrização denominada de parametrização local. Neste método, o parâmetro de continuação inicialmente escolhido é o fator de

A. Bonini Neto, Departamento de Engenharia de Biossistemas, UNESP, Tupã, Brasil,bonini@tupa.unesp.br

D. A. Alves, Departamento de Engenharia Elétrica, UNESP, Ilha Solteira, Brasil, dalves@dee.feis.unesp.br carregamento do sistema $(\lambda)$. Posteriormente, próximo ao PMC, efetua-se a troca, sendo que a variável escolhida como novo parâmetro é aquela que apresentar a maior variação no vetor tangente. Em geral, ou magnitude da tensão nodal $\left(\mathrm{V}_{\mathrm{k}}\right)$ ou ângulo da tensão nodal $\left(\theta_{\mathrm{k}}\right)$ de uma barra $\mathrm{k}$ qualquer é escolhida como novo parâmetro da continuação. Em [2], também foi apresentada uma técnica de parametrização local para o método da continuação envolvendo equações de retas no plano cartesiano fator de carregamento $(\lambda)$ e magnitude da tensão nodal $\left(\mathrm{V}_{\mathrm{k}}\right), \lambda-\mathrm{V}_{\mathrm{k}}$. A singularidade da matriz $\mathbf{J}$ é eliminada pela equação de uma reta passando por esse plano. Com isso, ao se incrementar o coeficiente angular dessa reta, obtém-se as sucessivas soluções do fluxo de carga. Uma ideia similar foi apresentada em [3], a diferença é que o plano para obtenção das curvas $\mathrm{P}-\mathrm{V}$ é formado pelo fator de carregamento $(\lambda)$ e o ângulo da tensão nodal $\left(\theta_{\mathrm{k}}\right), \lambda-\theta_{\mathrm{k}}$. Em [4] apresentou-se uma técnica global de parametrização geométrica que possibilita o traçado completo das curvas $\mathrm{P}-\mathrm{V}$ sem os problemas de mau-condicionamento. Na técnica, que associa a robustez com a simplicidade, a singularidade da matriz J é eliminada pela adição da equação de uma reta que passa por um ponto no plano formado pelas variáveis fator de carregamento $(\lambda)$ e a perda total de potência ativa $(P a)$. Em [5] apresenta-se uma eficiente técnica global de parametrização geométrica para o fluxo de carga continuado na qual a singularidade da matriz $\mathbf{J}$ é eliminada pela adição de uma equação da reta que passa através dos pontos no plano determinado pelas variáveis fator de carregamento $(\lambda)$ e soma de todas as magnitudes das tensões nodais $\left(\Sigma \mathrm{V}_{\mathrm{k}}\right)$, plano $\lambda$ $\Sigma \mathrm{V}_{\mathrm{k}}$, ou de todos os ângulos nodais $\left(\Sigma \theta_{\mathrm{k}}\right)$, plano $\lambda-\Sigma \theta_{\mathrm{k}}$. E por fim, em [6] utiliza-se a equação quadrática no plano formado pelas variáveis $\lambda-P a$. As vantagens e particularidades de cada uma dessas técnicas podem ser verificadas nas respectivas referências. $\mathrm{Na}$ literatura existem diversas outras propostas para o método de fluxo de carga continuado [7-12].

O objetivo nesta primeira parte do trabalho, foi apresentar uma modelagem matemática dos métodos aqui citados, ou seja, apresentar um fluxograma para obtenção dos determinantes das matrizes Jacobianas modificadas de sistemas de grande porte e também apresentar uma análise da matriz Jacobiana dos métodos de fluxo de carga continuado, uma vez que para cada método ocorrem modificações na matriz Jacobiana (J) convencional em consequência das reparametrizações necessárias para se eliminar os problemas de singularidade de $\mathbf{J}$ no PMC. 


\section{MATRIZ JACOBIANA do FluXo DE CARGA CONTINUADO}

As equações convencionais do fluxo de carga são:

$$
\begin{aligned}
& \mathbf{G}(\boldsymbol{\theta}, \mathbf{V})=\mathbf{0}, \text { ou } \\
& \Delta \mathbf{P}=\mathbf{P}^{\operatorname{esp}}-\mathbf{P}(\boldsymbol{\theta}, \mathbf{V})=\mathbf{0} \\
& \Delta \mathbf{Q}=\mathbf{Q}^{\operatorname{esp}}-\mathbf{Q}(\boldsymbol{\theta}, \mathbf{V})=\mathbf{0}
\end{aligned}
$$

sendo

$$
\begin{aligned}
& \mathrm{P}_{\mathrm{k}}(\boldsymbol{\theta}, \mathbf{V})=V_{k} \sum_{m \in K} V_{m}\left(G_{k m} \cos \theta_{k m}+B_{k m} \sin \theta_{k m}\right) \\
& \mathrm{Q}_{\mathrm{k}}(\boldsymbol{\theta}, \mathbf{V})=V_{k} \sum_{m \in \kappa} V_{m}\left(G_{k m} \sin \theta_{k m}-B_{k m} \cos \theta_{k m}\right)
\end{aligned}
$$

e $\boldsymbol{\theta}$ e $\mathbf{V}$ são os respectivos vetores de ângulo de fase e magnitude de tensão nodal; $\mathbf{P}^{\text {esp }}$ é o vetor da diferença entre os vetores de potência ativa, gerada $\left(\mathbf{P}_{\mathbf{g}}{ }^{\text {esp }}\right)$ e consumida $\left(\mathbf{P}_{\mathbf{c}}{ }^{\text {esp }}\right)$, especificada para as barras de carga $(P Q)$ e geração $(P V)$; e $\mathbf{Q} \mathbf{c}^{\text {esp }}$ é o vetor de potência reativa consumida especificada para as barras $P Q$.

A equação (1) assume que o carregamento da rede é proporcional ao do caso base e considera o fator de potência constante. $\mathbf{P}^{\text {esp }}$ e $\mathbf{Q}^{\text {esp }}$ também pode ser definido como sendo igual a $\left(\mathbf{k}_{\mathrm{Pg}} \mathbf{P}_{\mathrm{g}}{ }^{\text {esp }}+\mathbf{k}_{\mathrm{Pc}} \mathbf{P}_{\mathbf{c}}{ }^{\text {esp }}\right)$ e $\mathbf{k}_{\mathrm{Qc}} \mathbf{Q}_{\mathrm{c}}{ }^{\text {esp }}$, respectivamente. Os vetores $\mathbf{k}_{\mathrm{Pg}}$, $\mathbf{k}_{\mathrm{Pc}}$ e $\mathbf{k}_{\mathrm{Qc}}$ são parâmetros fixos usados para caracterizar um específico cenário de carga.

A resolução do sistema de equações (1) pelo método de Newton é feita através da linearização da função $\mathbf{G}(\mathbf{x})$, em que $\mathbf{G}(\mathbf{x})=[\Delta \mathbf{P}, \Delta \mathbf{Q}]^{\prime}=\left[\mathrm{G}_{1}, \mathrm{G}_{2}, \ldots\right]^{\prime}$ e $\mathbf{x}=[\boldsymbol{\theta}, \mathbf{V}]$, para $\mathbf{x}$ $=\mathbf{x}^{\mathbf{i}}$ considerando os dois primeiros termos da série de Taylor:

$$
G\left(x^{i}+\Delta x^{i}\right) \cong G\left(x^{i}\right)+J\left(x^{i}\right) \Delta x^{i}
$$

sendo a matriz jacobiana $\mathbf{J}$ dada por:

$$
\mathbf{J}=\frac{\partial \mathbf{G}}{\partial \mathbf{x}}=\left[\begin{array}{cccc}
\frac{\partial \mathrm{G}_{1}}{\partial \mathrm{x}_{1}} & \frac{\partial \mathrm{G}_{1}}{\partial \mathrm{x}_{2}} & \ldots & \frac{\partial \mathrm{G}_{1}}{\partial \mathrm{x}_{\mathrm{n}}} \\
\frac{\partial \mathrm{G}_{2}}{\partial \mathrm{x}_{1}} & \frac{\partial \mathrm{G}_{2}}{\partial \mathrm{x}_{2}} & \ldots & \frac{\partial \mathrm{G}_{2}}{\partial \mathrm{x}_{\mathrm{n}}} \\
\cdots & \ldots & \ldots & \ldots \\
\frac{\partial \mathrm{G}_{\mathrm{n}}}{\partial \mathrm{x}_{1}} & \frac{\partial \mathrm{G}_{\mathrm{n}}}{\partial \mathrm{x}_{2}} & \ldots & \frac{\partial \mathrm{G}_{\mathrm{n}}}{\partial \mathrm{x}_{\mathrm{n}}}
\end{array}\right]
$$

O vetor de correção $\Delta \mathbf{x}$ é calculado impondo-se que:

$$
\mathbf{G}\left(\mathbf{x}^{\mathbf{i}}\right)+\mathbf{J}\left(\mathbf{x}^{\mathbf{i}}\right) \Delta \mathbf{x}^{\mathbf{i}}=\mathbf{0}
$$

que é a maneira linearizada de se resolver o problema $\mathbf{G}\left(\mathbf{x}^{\mathbf{i}}+\Delta \mathbf{x}^{\mathbf{i}}\right)=\mathbf{0}$. No caso em que $n=2$, a equação (3) assume a forma:

$$
\begin{aligned}
& \mathrm{G}_{1}\left(\mathrm{x}_{1}, \mathrm{x}_{2}\right) \cong \mathrm{G}_{1}\left(\mathrm{x}_{1}{ }^{\mathrm{i}}, \mathrm{x}_{2}{ }^{\mathrm{i}}\right)+\left.\frac{\partial \mathrm{G}_{1}}{\partial \mathrm{x}_{1}}\right|_{\mathrm{i}} \Delta \mathrm{x}_{1}{ }^{(\mathrm{i})}+\left.\frac{\partial \mathrm{G}_{1}}{\partial \mathrm{x}_{2}}\right|_{\mathrm{i}} \Delta \mathrm{x}_{2}{ }^{(\mathrm{i})} \\
& \mathrm{G}_{2}\left(\mathrm{x}_{1}, \mathrm{x}_{2}\right) \cong \mathrm{G}_{2}\left(\mathrm{x}_{1}{ }^{\mathrm{i}}, \mathrm{x}_{2}{ }^{\mathrm{i}}\right)+\left.\frac{\partial \mathrm{G}_{2}}{\partial \mathrm{x}_{1}}\right|_{\mathrm{i}} \Delta \mathrm{x}_{1}{ }^{(\mathrm{i})}+\left.\frac{\partial \mathrm{G}_{2}}{\partial \mathrm{x}_{2}}\right|_{\mathrm{i}} \Delta \mathrm{x}_{2}{ }^{(\mathrm{i})}
\end{aligned}
$$

e na forma matricial representa:

$$
\left[\begin{array}{l}
\mathrm{G}_{1}\left(\mathrm{x}_{1}{ }^{\mathrm{i}}, \mathrm{x}_{2}{ }^{\mathrm{i}}\right) \\
\mathrm{G}_{2}\left(\mathrm{x}_{1}{ }^{\mathrm{i}}, \mathrm{x}_{2}{ }^{\mathrm{i}}\right)
\end{array}\right]+\left[\begin{array}{ll}
\frac{\partial \mathrm{G}_{1}}{\partial \mathrm{x}_{1}} & \frac{\partial \mathrm{G}_{1}}{\partial \mathrm{x}_{2}} \\
\frac{\partial \mathrm{G}_{2}}{\partial \mathrm{x}_{1}} & \frac{\partial \mathrm{G}_{2}}{\partial \mathrm{x}_{2}}
\end{array}\right]^{(\mathrm{i})} \cdot\left[\begin{array}{c}
\Delta \mathrm{x}_{1}{ }^{(i)} \\
\Delta \mathrm{x}_{2}{ }^{(i)}
\end{array}\right]=\left[\begin{array}{l}
0 \\
0
\end{array}\right]
$$

A nova solução $\mathbf{x}^{(\mathbf{i}+1)}$ é:

$\mathrm{e}$

$$
\mathbf{x}^{i+1}=\mathbf{x}^{i}+\Delta \mathbf{x}^{i}
$$

$$
\Delta \mathbf{x}^{\mathbf{i}}=-\left[\mathbf{J}\left(\mathbf{x}^{\mathbf{i}}\right)\right]^{-1} \mathbf{G}\left(\mathbf{x}^{\mathbf{i}}\right)
$$

Para o fluxo de carga continuado o sistema de equações (1) passa a ter uma variável a mais, ou seja, adiciona-se uma variável $\lambda$ que representada o fator de carregamento do sistema, tornando-se:

$$
\begin{aligned}
& \mathbf{G}(\boldsymbol{\theta}, \mathbf{V}, \lambda)=\mathbf{0}, \text { ou } \\
& \Delta \mathbf{P}=\lambda \mathbf{P}^{\mathbf{e s p}}-\mathbf{P}(\boldsymbol{\theta}, \mathbf{V})=\mathbf{0} \\
& \Delta \mathbf{Q}=\lambda \mathbf{Q}^{\mathbf{e s p}}-\mathbf{Q}(\boldsymbol{\theta}, \mathbf{V})=\mathbf{0}
\end{aligned}
$$

$\mathrm{O}$ traçado da curva $\mathrm{P}-\mathrm{V}$ pode ser feito por sucessivas soluções de (10), utilizando um FC. Adotando $\lambda$ como parâmetro obtém-se o PMC. A partir do caso base $(\lambda=1)$, seu valor é aumentado gradualmente até um valor para o qual nenhuma solução seja obtida, ou seja, para qual o processo iterativo do fluxo de potência não convirja ou divirja. Em geral, neste ponto, haverá um controle de passo consistindo de uma simples redução no incremento (tamanho do passo) de $\lambda$ e a nova solução é obtida a partir da última solução convergente. Considera-se o PMC como o último ponto convergido após várias repetições desse procedimento. No entanto, a divergência do $\mathrm{FC}$ é uma consequência da singularidade da matriz Jacobiana (J) de (10) no PMC e, portanto, seu valor não pode ser determinado com precisão. A fim de eliminar as dificuldades numéricas resultantes da singularidade de $\mathbf{J}$ e permitir a determinação do PMC, foram propostas diferentes parametrizações para o método da continuação [1-13]. Como o sistema apresenta uma variável a mais do que equação, é necessário acrescentar mais uma equação. Logo, a partir da equação (10) cada método apresenta a sua particularidade. 


\section{FluXOGRAMA PARA OBTENÇÃO DOS DETERMINANTES DE SISTEMAS DE GRANDE PORTE}

Uma das alternativas de verificar a singularidade da matriz $\mathbf{J}$ é através da inversão do sinal do determinante no PMC, ou seja, o determinante é nulo exatamente no PMC, podendo ser verificado em sistemas de pequeno porte por meio do gráfico dos valores numéricos do determinante em função do carregamento $\lambda$. A dimensão da matriz quadrada $\mathbf{J}$ é da ordem de $\left(2 n_{P Q}+n_{P V}\right)$, sendo que $n_{P Q}$ e $n_{P V}$ correspondem ao número de barras PQ e PV, respectivamente. Então, por exemplo, para um sistema como o IEEE-14 barras que é representado por 9 barras de carga, 5 barras de geração e 20 linhas de transmissão (LT), a matriz $\mathbf{J}$ convencional é da ordem de $23 \times 23(2 \times 9+5)$. Sistemas de grande porte como o IEEE-300 barras, duas configurações do sistema Sul-Sudeste Brasileiro de 638 e 787 barras e um sistema de 904 barras localizado no Sudoeste Americano podem apresentar matrizes Jacobianas da ordem $530 \times 530, \quad 1189 \times 1189, \quad 1461 \times 1461$ e $1675 \times 1675$ respectivamente, acarretando em valores muito altos para os determinantes, maiores do que $1 \mathrm{e}+309$.

Determinantes dessa ordem são representados no MATLAB [16] por meio do símbolo inf ( $\infty$, infinito). Como alternativa para visualizar graficamente os seus valores efetuase a sua redução através da multiplicação dos elementos da matriz por $1 / n$, em que $n$ neste caso é igual a 10 para o sistema IEEE-300 barras e igual a 100 para o sistema de 638 e 787 barras. Outros valores podem ser utilizados.

Estudos envolvendo estas análises dos determinantes foram iniciados em [17], porém, para sistemas maiores do que o IEEE-300 barras, não foram possíveis a sua obtenção, principalmente a parte gráfica. A Fig. 1 a seguir representa o fluxograma desse procedimento para obtenção dos determinantes de sistemas de grande porte, em que $m$ é a ordem da matriz $\mathbf{J}$.

Para o sistema de 638 barras, por exemplo, os determinantes atingiram valores em torno de $10^{2084}$. Essa análise do valor e também para o esboço dos gráficos, só foram possíveis de obter, com a utilização da metodologia apresentada pelo fluxograma na Fig. 1. Os resultados são apresentados na segunda parte deste trabalho.

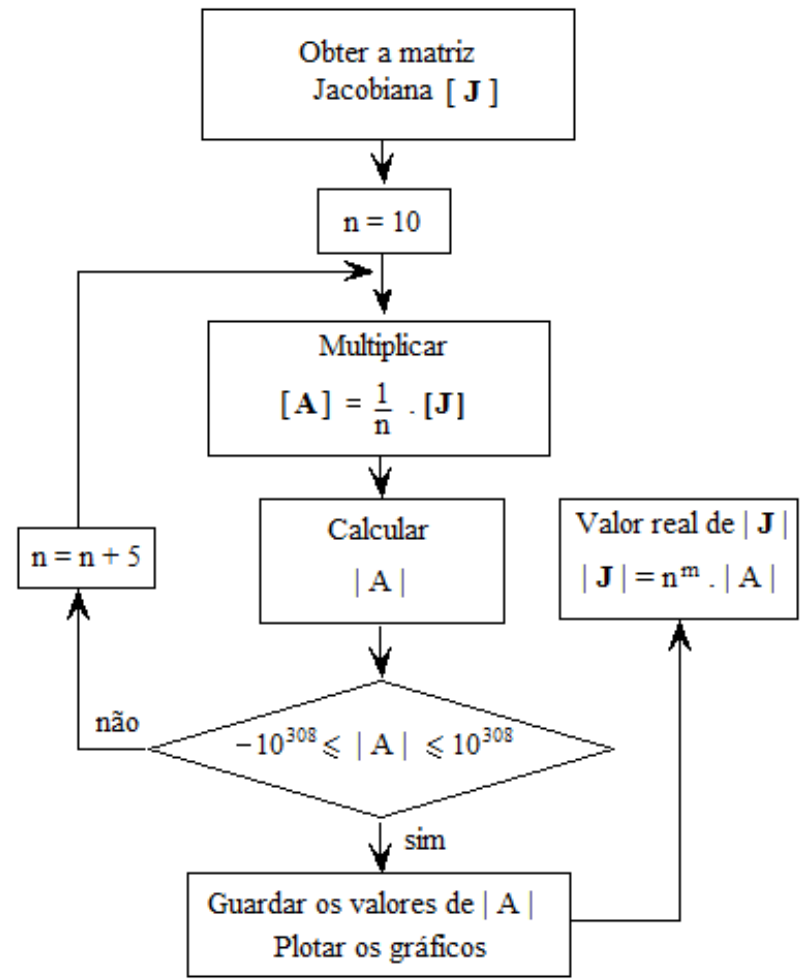

Figura 1.Fluxograma para obtenção dos valores dos determinantes de sistemas de grande porte.

O fluxograma apresentado na Fig. 1 corresponde ao seguinte algoritmo.

i. Obter a matriz Jacobiana $\mathbf{J}$;

ii. $\operatorname{Adotar} \mathrm{n}=10$;

iii. Multiplicar a matriz $\mathbf{J}$ por $1 / \mathrm{n}$ obtendo $\mathbf{A}$;

iv. $\mathrm{Se}-10^{308} \leq|\mathrm{A}| \leq 10^{308}$.

Guarda os valores de $|\mathrm{A}|$.

Plota os gráficos dos determinantes.

Valor real de $|\mathrm{J}|=\mathrm{n}^{\mathrm{m}} \cdot|\mathrm{A}|$.

Caso contrário;

v. $n=n+5$ e volte ao passo iii.

\section{ANÁliSE DA MATRIZ JACOBIANA MODIFICADA DO FLUXO DE CARGA CONTINUADO}

A equação $\mathrm{E}(\boldsymbol{\theta}, \mathbf{V}, \lambda)$ foi acrescentada ao sistema de equações (10) no método proposto em [1], passando a ser:

$$
\left\{\begin{array}{l}
\Delta \mathbf{P}=\lambda \mathbf{P}^{\mathbf{e s p}}-\mathbf{P}(\boldsymbol{\theta}, \mathbf{V})=\mathbf{0} \\
\boldsymbol{\Delta} \mathbf{Q}=\lambda \mathbf{Q}^{\mathbf{e s p}}-\mathbf{Q}(\boldsymbol{\theta}, \mathbf{V})=\mathbf{0} \\
\mathrm{E}(\boldsymbol{\theta}, \mathbf{V}, \lambda)=\left(\lambda-\lambda^{0}\right) \text { ou }\left(\mathrm{V}_{\mathrm{k}}-\mathrm{V}_{\mathrm{k}}^{0}\right) \text { ou }\left(\theta_{\mathrm{k}}-\theta_{\mathrm{k}}^{0}\right)=0
\end{array}\right.
$$

Linearizando pelos dois primeiros termos da série de Taylor: 


$$
\begin{aligned}
& \Delta \mathbf{P}+\frac{\partial \Delta \mathbf{P}}{\partial \boldsymbol{\theta}} \Delta \boldsymbol{\theta}+\frac{\partial \Delta \mathbf{P}}{\partial \mathbf{V}} \Delta \mathbf{V}+\frac{\partial \Delta \mathbf{P}}{\partial \lambda} \Delta \lambda=0 \\
& \Delta \mathbf{Q}+\frac{\partial \Delta \mathbf{Q}}{\partial \boldsymbol{\theta}} \Delta \boldsymbol{\theta}+\frac{\partial \Delta \mathbf{Q}}{\partial \mathbf{V}} \Delta \mathbf{V}+\frac{\partial \Delta \mathbf{Q}}{\partial \lambda} \Delta \lambda=0 \\
& \mathrm{E}+\frac{\partial \mathrm{E}}{\partial \boldsymbol{\theta}} \boldsymbol{\Delta} \boldsymbol{\theta}+\frac{\partial \mathrm{E}}{\partial \mathbf{V}} \Delta \mathbf{V}+\frac{\partial \mathrm{E}}{\partial \lambda} \Delta \lambda=0
\end{aligned}
$$

e que na forma matricial torna-se:

$$
\left[\begin{array}{c}
\Delta \mathbf{P} \\
\Delta \mathbf{Q} \\
\mathrm{E}
\end{array}\right]+\left[\begin{array}{ccc}
\frac{\partial \Delta \mathbf{P}}{\partial \boldsymbol{\theta}} & \frac{\partial \Delta \mathbf{P}}{\partial \mathbf{V}} & \frac{\partial \Delta \mathbf{P}}{\partial \lambda} \\
\frac{\partial \boldsymbol{\Delta} \mathbf{Q}}{\partial \boldsymbol{\theta}} & \frac{\partial \Delta \mathbf{Q}}{\partial \mathbf{V}} & \frac{\partial \Delta \mathbf{Q}}{\partial \lambda} \\
\frac{\partial \mathrm{E}}{\partial \boldsymbol{\theta}} & \frac{\partial \mathrm{E}}{\partial \mathbf{V}} & \frac{\partial \mathrm{E}}{\partial \lambda}
\end{array}\right]\left[\begin{array}{c}
\Delta \boldsymbol{\theta} \\
\Delta \mathbf{V} \\
\Delta \lambda
\end{array}\right]=\mathbf{0}
$$

ou

$$
\left[\begin{array}{c}
\Delta \mathbf{P} \\
\Delta \mathbf{Q} \\
\mathrm{E}
\end{array}\right]+\left[\begin{array}{ccc}
-\frac{\partial \mathbf{P}}{\partial \boldsymbol{\theta}} & -\frac{\partial \mathbf{P}}{\partial \mathbf{V}} & \mathbf{P}^{\mathbf{e s p}} \\
-\frac{\partial \mathbf{Q}}{\partial \boldsymbol{\theta}} & -\frac{\partial \mathbf{Q}}{\partial \mathbf{V}} & \mathbf{Q}^{\mathbf{e s p}} \\
\frac{\partial \mathrm{E}}{\partial \boldsymbol{\theta}} & \frac{\partial \mathrm{E}}{\partial \mathbf{V}} & \frac{\partial \mathrm{E}}{\partial \lambda}
\end{array}\right]\left[\begin{array}{c}
\Delta \boldsymbol{\theta} \\
\Delta \mathbf{V} \\
\Delta \lambda
\end{array}\right]=\mathbf{0}
$$

Caso o parâmetro for a variável $\lambda$ (Fig. 2(a)), então se utiliza $\mathrm{E}(\boldsymbol{\theta}, \mathbf{V}, \lambda)=\left(\lambda-\lambda^{0}\right)=0$, e nesse caso:

$$
\left[\begin{array}{c}
\Delta \mathbf{P} \\
\Delta \mathbf{Q} \\
\mathrm{E}
\end{array}\right]+\left[\begin{array}{ccc}
-\frac{\partial \mathbf{P}}{\partial \boldsymbol{\theta}} & -\frac{\partial \mathbf{P}}{\partial \mathbf{V}} & \mathbf{P}^{\mathbf{e s p}} \\
-\frac{\partial \mathbf{Q}}{\partial \boldsymbol{\theta}} & -\frac{\partial \mathbf{Q}}{\partial \mathbf{V}} & \mathbf{Q}^{\mathbf{e s p}} \\
\mathbf{0} & \mathbf{0} & 1
\end{array}\right]\left[\begin{array}{c}
\Delta \boldsymbol{\theta} \\
\Delta \mathbf{V} \\
\Delta \lambda
\end{array}\right]=\mathbf{0}
$$

ou

$$
-\left[\begin{array}{ccc}
-\mathbf{J} & \mathbf{P}^{\mathbf{e s p}} \\
\mathbf{0} & \mathbf{0} & 1
\end{array}\right]\left[\begin{array}{c}
\Delta \mathbf{x} \\
\Delta \lambda
\end{array}\right]=-\mathbf{J}_{\mathbf{m}}\left[\begin{array}{c}
\Delta \mathbf{x} \\
\Delta \lambda
\end{array}\right]=\left[\begin{array}{c}
\Delta \mathbf{G} \\
\mathrm{E}
\end{array}\right]
$$

em que $\mathbf{J}_{\mathbf{m}}$ é dada por:

$$
\left[\begin{array}{ccc} 
& & \mathbf{P}^{\mathbf{e s p}} \\
\mathbf{0} & \mathbf{0} & \mathbf{Q}^{\text {esp }}
\end{array}\right]
$$

ou por:

$$
\left[\begin{array}{ccc}
-\mathbf{J} & \mathbf{P}^{\mathbf{e s p}} \\
{\left[0,0, . ., 1_{\mathrm{k}}, \ldots, 0\right]} & \mathbf{0} & 0
\end{array}\right] \text { ou }\left[\begin{array}{ccc}
\mathbf{Q}^{\mathbf{e s p}} & -\mathbf{J} & \mathbf{P}^{\mathbf{e s p}} \\
\mathbf{0} & {\left[0,0, . ., 1_{\mathrm{k}}, \ldots, 0\right]} & \mathbf{Q}^{\mathbf{e s p}} \\
0
\end{array}\right]
$$

caso a variável escolhida como parâmetro seja $\theta_{\mathrm{k}}$ $\left(\mathrm{E}(\boldsymbol{\theta}, \mathbf{V}, \lambda)=\left(\theta_{\mathrm{k}}-\theta_{\mathrm{k}}^{0}\right)=0\right)$ ou $\mathrm{V}_{\mathrm{k}}\left(\mathrm{E}(\boldsymbol{\theta}, \mathbf{V}, \lambda)=\left(\mathrm{V}_{\mathrm{k}}-\mathrm{V}_{\mathrm{k}}^{0}\right)=0\right)$, ver Fig. 2(b).
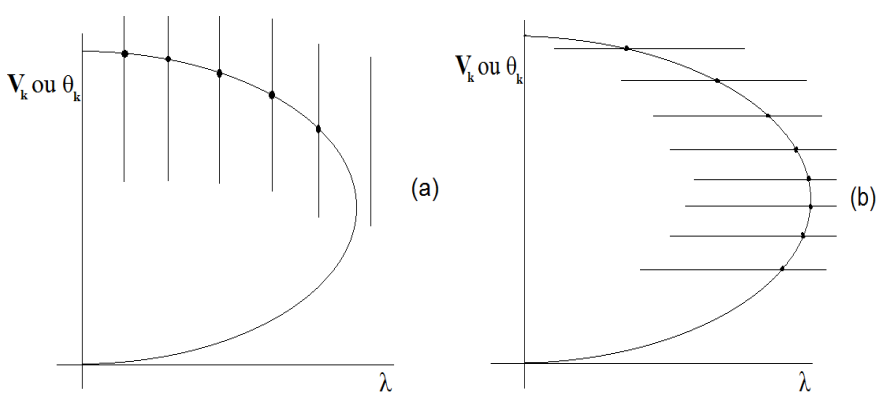

Figura 2. Método da parametrização local: (a) $\lambda$ como parâmetro, (b) $V_{k}$ ou $\theta_{k}$ como parâmetro.

No método apresentado em [2] acrescentou-se ao sistema de equações (10) a equação de uma reta $\mathrm{W}$ :

$$
\left\{\begin{array}{l}
\Delta \mathbf{P}=\lambda \mathbf{P}^{\mathbf{e s p}}-\mathbf{P}(\boldsymbol{\theta}, \mathbf{V})=\mathbf{0} \\
\Delta \mathbf{Q}=\lambda \mathbf{Q}^{\mathbf{e s p}}-\mathbf{Q}(\boldsymbol{\theta}, \mathbf{V})=\mathbf{0} \\
\mathrm{W}(\boldsymbol{\theta}, \mathbf{V}, \lambda, \alpha)=\alpha\left(\lambda-\lambda^{0}\right)-\left(\mathrm{V}_{\mathrm{k}}-\mathrm{V}_{\mathrm{k}}^{0}\right)=0
\end{array}\right.
$$

sendo $\alpha$ o coeficiente angular da reta, e $\lambda^{0}$ e $V_{k}^{0}$ os pontos escolhidos no plano representando o centro do feixe de retas inicial, Fig. 3(a), os quais podem ser alterados.

Linearizando a equação (19) pelos dois primeiros termos da série de Taylor, tem-se:

$$
\begin{aligned}
& \Delta \mathbf{P}+\frac{\partial \Delta \mathbf{P}}{\partial \boldsymbol{\theta}} \Delta \boldsymbol{\theta}+\frac{\partial \Delta \mathbf{P}}{\partial \mathbf{V}} \Delta \mathbf{V}+\frac{\partial \Delta \mathbf{P}}{\partial \lambda} \Delta \lambda=0 \\
& \Delta \mathbf{Q}+\frac{\partial \Delta \mathbf{Q}}{\partial \boldsymbol{\theta}} \Delta \boldsymbol{\theta}+\frac{\partial \Delta \mathbf{Q}}{\partial \mathbf{V}} \Delta \mathbf{V}+\frac{\partial \Delta \mathbf{Q}}{\partial \lambda} \Delta \lambda=0 \\
& \mathrm{~W}+\frac{\partial \mathrm{W}}{\partial \boldsymbol{\theta}} \boldsymbol{\Delta} \boldsymbol{\theta}+\frac{\partial \mathrm{W}}{\partial \mathbf{V}} \boldsymbol{\Delta} \mathbf{V}+\frac{\partial \mathrm{W}}{\partial \lambda} \Delta \lambda=0
\end{aligned}
$$

e que na forma matricial torna-se:

$$
\left[\begin{array}{c}
\boldsymbol{\Delta} \mathbf{P} \\
\boldsymbol{\Delta} \mathbf{Q} \\
\mathrm{W}
\end{array}\right]+\left[\begin{array}{ccc}
\frac{\partial \Delta \mathbf{P}}{\partial \boldsymbol{\theta}} & \frac{\partial \Delta \mathbf{P}}{\partial \mathbf{V}} & \frac{\partial \Delta \mathbf{P}}{\partial \lambda} \\
\frac{\partial \boldsymbol{\Delta} \mathbf{Q}}{\partial \boldsymbol{\theta}} & \frac{\partial \Delta \mathbf{Q}}{\partial \mathbf{V}} & \frac{\partial \Delta \mathbf{Q}}{\partial \lambda} \\
\frac{\partial \mathrm{W}}{\partial \boldsymbol{\theta}} & \frac{\partial \mathrm{W}}{\partial \mathbf{V}} & \frac{\partial \mathrm{W}}{\partial \lambda}
\end{array}\right]\left[\begin{array}{c}
\boldsymbol{\Delta} \boldsymbol{\theta} \\
\boldsymbol{\Delta} \mathbf{V} \\
\Delta \lambda
\end{array}\right]=\mathbf{0}
$$

ou

$$
\left[\begin{array}{c}
\Delta \mathbf{P} \\
\Delta \mathbf{Q} \\
\mathrm{W}
\end{array}\right]+\left[\begin{array}{ccc}
-\frac{\partial \mathbf{P}}{\partial \boldsymbol{\theta}} & -\frac{\partial \mathbf{P}}{\partial \mathbf{V}} & \mathbf{P}^{\mathbf{e s p}} \\
-\frac{\partial \mathbf{Q}}{\partial \boldsymbol{\theta}} & -\frac{\partial \mathbf{Q}}{\partial \mathbf{V}} & \mathbf{Q}^{\mathbf{e s p}} \\
\mathbf{0} & -\left[0,0, . ., 1_{\mathrm{k}}, \ldots, 0\right] & \alpha
\end{array}\right]\left[\begin{array}{c}
\Delta \boldsymbol{\theta} \\
\boldsymbol{\Delta} \mathbf{V} \\
\Delta \lambda
\end{array}\right]=\mathbf{0}
$$

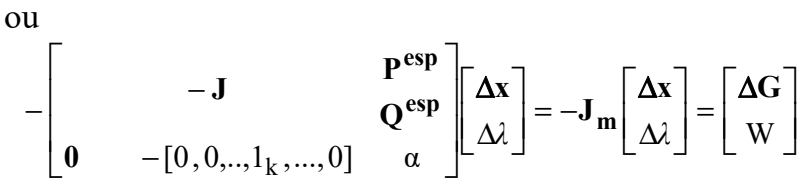




$$
\mathbf{J}_{\mathbf{m}}=\left[\begin{array}{ccc}
-\mathbf{J} & & \mathbf{P}^{\text {esp }} \\
& & \mathbf{Q}^{\text {esp }} \\
-\left[0,0, . ., 1_{\mathrm{k}}, \ldots, 0\right] & \mathbf{0} & \alpha
\end{array}\right]
$$

Formulações similares foram obtidas em [3], a diferença é que o plano utilizado para obtenção da curva $P-V$ é o $\lambda-\theta_{\mathrm{k}}$, ver Fig. 3(a). Nesse caso se acrescentou ao sistema de equações (10), a equação da seguinte reta R:

$$
\mathrm{R}(\boldsymbol{\theta}, \mathbf{V}, \lambda, \alpha)=\alpha\left(\lambda-\lambda^{0}\right)-\left(\theta_{\mathrm{k}}-\theta_{\mathrm{k}}^{0}\right)=0
$$

obtendo-se assim a matriz $\mathbf{J}_{\mathbf{m}}$ :

$$
\mathbf{J}_{\mathbf{m}}=\left[\begin{array}{ccc}
-\mathbf{J} & & \mathbf{P}^{\text {esp }} \\
-\left[0,0, . ., 1_{\mathrm{k}}, \ldots, 0\right] & \mathbf{0} & \alpha
\end{array}\right]
$$

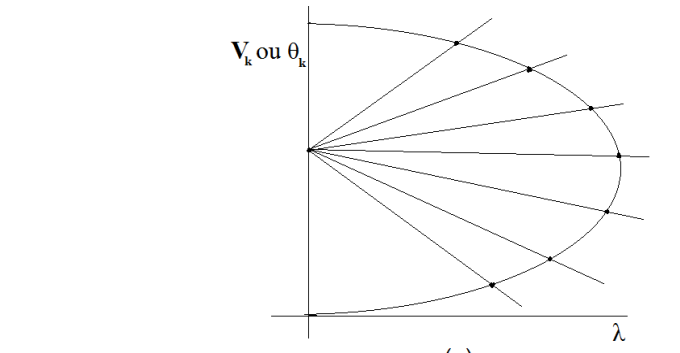

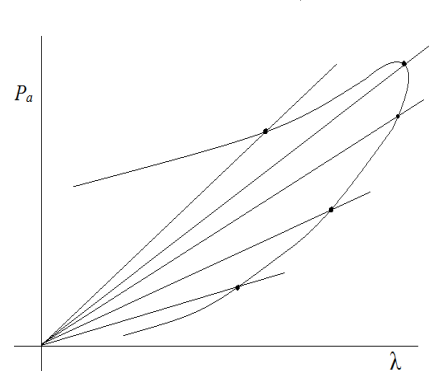

(b)

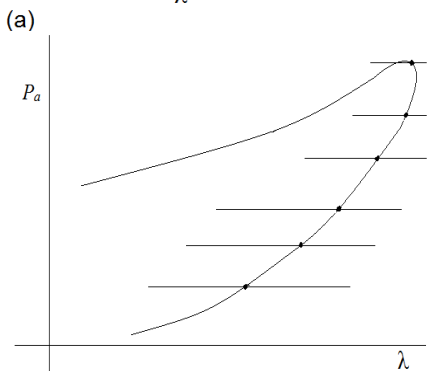

(c)
Figura 3. Métodos: (a) parametrizado por $\alpha$ no plano $\lambda-V_{\mathrm{k}}$ ou $\lambda-\theta_{\mathrm{k}}$, (b) parametrizado por $\alpha$ no plano $\lambda-P a$, (c) parametrizado por $P a$.

Já no método proposto em [4], que é uma parametrização global, a equação acrescida à equação (10) é a seguinte:

$$
\mathrm{T}(\boldsymbol{\theta}, \mathbf{V}, \lambda, \alpha)=\alpha\left(\lambda-\lambda^{0}\right)-\left[P a(\boldsymbol{\theta}, \mathbf{V})-\mathrm{Pa}^{0}\right]=0
$$

sendo

$$
\operatorname{Pa}(\boldsymbol{\theta}, \mathbf{V})=\sum_{\mathrm{i} \text { e } \in \mathrm{j} \Omega} \mathrm{g}_{\mathrm{ij}}\left[\mathrm{V}_{\mathrm{i}}^{2}+\mathrm{V}_{\mathrm{j}}^{2}-2 \mathrm{~V}_{\mathrm{i}} \mathrm{V}_{\mathrm{j}} \cos \left(\theta_{\mathrm{i}}-\theta_{\mathrm{j}}\right)\right]
$$

e $\alpha$ o coeficiente angular da reta (parâmetro utilizado). $P a$ representa a perda total de potência ativa, por isso trata-se de uma técnica de parametrização global, ver Fig. 3(b). Caso $\alpha$ $=0$ e variando-se o valor de $P a^{0}$ a solução do sistema (29) resultará em $\mathrm{Pa}$ como parâmetro [14-15], Fig. 3(c). Linearizando pelos dois primeiros termos da série de Taylor, obtém-se o sistema:

$$
-\left[\begin{array}{ccc}
-\mathbf{J} & & \mathbf{P}^{\text {esp }} \\
\frac{\partial \mathrm{T}}{\partial \boldsymbol{\theta}} & \frac{\partial \mathrm{T}}{\partial \mathbf{V}} & \alpha
\end{array}\right]\left[\begin{array}{c}
\Delta \mathbf{x} \\
\Delta \lambda
\end{array}\right]=-\mathbf{J}_{\mathbf{m}}\left[\begin{array}{c}
\Delta \mathbf{x} \\
\Delta \lambda
\end{array}\right]=\left[\begin{array}{c}
\Delta \mathbf{G} \\
\mathrm{T}
\end{array}\right]
$$

no qual a matriz $\mathbf{J}_{\mathbf{m}}$ é dada por:

$$
\mathbf{J}_{\mathbf{m}}=\left[\begin{array}{ccc} 
& & \mathbf{P}^{\text {esp }} \\
-\mathbf{J} & & \mathbf{Q}^{\text {esp }} \\
\frac{\partial \mathrm{T}}{\partial \boldsymbol{\theta}} & \frac{\partial \mathrm{T}}{\partial \mathbf{V}} & \alpha
\end{array}\right]
$$

Outra técnica de parametrização global foi proposta em [5], em que acrescenta-se ao sistema de equações (10) a seguinte equação:

$$
\mathbf{S}(\boldsymbol{\theta}, \mathbf{V}, \lambda, \alpha)=\alpha\left(\lambda-\lambda^{0}\right)-\left(\frac{\mathbf{Y}}{\mu}-\frac{\mathbf{Y}^{0}}{\mu}\right)=0
$$

sendo $\mu$ o número de barras do sistema e $\mathbf{Y}=\sum_{\mathrm{i}=1}^{\mu} \mathrm{V}_{\mathrm{i}}$ ou $\mathbf{Y}=\sum_{\mathrm{i}=1}^{\mu} \theta_{\mathrm{i}}$

Expandindo em série de Taylor, considerando $\mathbf{Y}=\sum_{\mathrm{i}=1}^{\mu} \mathrm{V}_{\mathrm{i}}$, tem-se o seguinte sistema de equações, que na forma matricial torna-se:

$$
-\left[\begin{array}{ccc}
-\mathbf{J} & \mathbf{P}^{\text {esp }} \\
& \mathbf{Q}^{\text {esp }} \\
\mathbf{0} & \frac{1}{\mu} & \alpha
\end{array}\right]\left[\begin{array}{l}
\Delta \mathbf{x} \\
\Delta \lambda
\end{array}\right]=-\mathbf{J}_{\mathbf{m}}\left[\begin{array}{c}
\Delta \mathbf{x} \\
\Delta \lambda
\end{array}\right]=\left[\begin{array}{c}
\Delta \mathbf{G} \\
\mathbf{S}
\end{array}\right]
$$

A matriz $\mathbf{J}_{\mathbf{m}}$ do método passa a ser dada por:

$$
\mathbf{J}_{\mathbf{m}}=\left[\begin{array}{ccc}
-\mathbf{J} & \mathbf{P}^{\text {esp }} \\
\mathbf{0} & \frac{1}{\mu} & \alpha
\end{array}\right]
$$

Já para $\mathbf{Y}=\sum_{\mathrm{i}=1}^{\mu} \theta_{\mathrm{i}}$, a matriz $\mathbf{J}_{\mathbf{m}}$ passa a ser dada por: 


$$
\mathbf{J}_{\mathbf{m}}=\left[\begin{array}{ccc}
-\mathbf{J} & \mathbf{P}^{\text {esp }} \\
\frac{1}{\mu} & \mathbf{0} & \alpha
\end{array}\right]
$$

A Fig. 4(a) apresenta a geometria da trajetória de soluções do método proposto em [5].
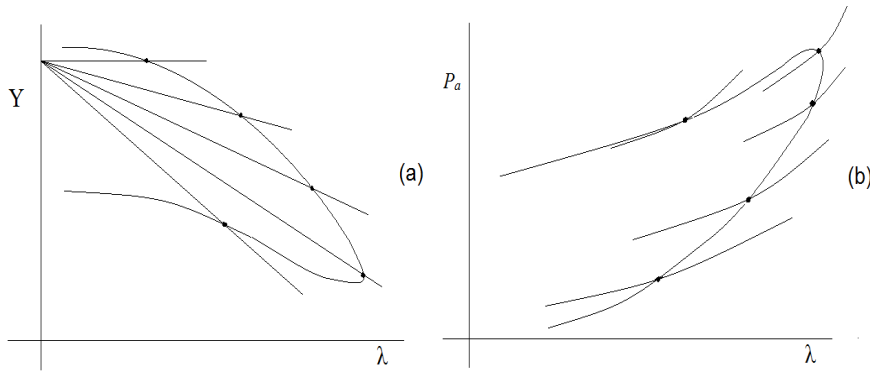

Figura 4: Métodos: (a) parametrizado pelo coeficiente angular da reta $\alpha$ no plano $\lambda-\mathbf{Y}$, (b) parametrizado pelo coeficiente $\alpha$ das equações das parábolas.

Em [6], a equação acrescida à equação (10) é dada por:

$$
\mathrm{M}(\boldsymbol{\theta}, \mathbf{V}, \boldsymbol{\lambda}, \boldsymbol{\alpha})=P a(\boldsymbol{\theta}, \mathbf{V}, \lambda)-\left[a(\lambda+\alpha)^{2}+b(\boldsymbol{\lambda}+\alpha)+c\right]=0
$$

sendo que $P a$, a perda total de potência ativa, é dada pela equação (28), enquanto que os coeficientes $a, b$ e $c$ são calculados através do desenvolvimento do produtório do coeficiente do polinômio interpolador de Lagrange para três pontos conhecidos do plano $\lambda-P a$. Após a determinação desses coeficientes, para cada valor atribuído ao parâmetro $\alpha$ define-se novas equações de parábolas, ver Fig. 4(b). Nota-se que neste caso, equação (35), $\alpha$ é o parâmetro que representa o coeficiente da equação da parábola e não mais da reta conforme foi apresentado nas equações (19), (25), (27) e (31). Nesse caso a matriz $\mathbf{J}_{\mathbf{m}}$ passa a ser dada por:

$$
\mathbf{J}_{\mathbf{m}}=\left[\begin{array}{ccc}
\mathbf{G}_{\boldsymbol{\theta}} & \mathbf{G}_{\mathbf{V}} & \mathbf{G}_{\lambda} \\
\partial P a / \partial \boldsymbol{\theta} & \partial P a / \partial \mathbf{V} & -(2 a(\lambda+\alpha)+b)
\end{array}\right]
$$

Para o cálculo do determinante, por exemplo, utilizando esta última equação (36) que representa a metodologia utilizando as parabolas no plano $\lambda-P a$, o determinante é calculado por meio da equação (37).

$$
\left|\mathbf{J}_{\mathbf{m}}\right|=\left|\begin{array}{ccc}
\frac{\partial \boldsymbol{\Delta} \mathbf{P}}{\partial \boldsymbol{\theta}} / n & \frac{\partial \boldsymbol{\Delta} \mathbf{P}}{\partial \mathbf{V}} / n & \frac{\partial \boldsymbol{\Delta} \mathbf{P}}{\partial \lambda} / n \\
\frac{\partial \boldsymbol{\Delta} \mathbf{Q}}{\partial \boldsymbol{\theta}} / n & \frac{\partial \boldsymbol{\Delta} \mathbf{Q}}{\partial \mathbf{V}} / n & \frac{\partial \boldsymbol{\Delta} \mathbf{Q}}{\partial \lambda} / n \\
\partial P a / \partial \boldsymbol{\theta}) / n & (\partial P a / \partial \mathbf{V}) / n & {\left[-\left(2 a\left(\lambda^{2}+\alpha\right)+b\right)\right] / \mathrm{n}}
\end{array}\right|
$$

\section{CONCLUSÃO}

Nesta primeira parte do artigo foi apresentado um fluxograma para obtenção dos determinantes da $\mathbf{J}_{\mathbf{m}}$ de sistemas de grande porte e uma análise da singularidade da matriz Jacobiana do fluxo de carga continuado para várias técnicas de parametrização geométrica visando a obtenção do PMC de sistemas elétricos de potência. Os parâmetros utilizados foram: o fator de carregamento $(\lambda)$, a magnitude de tensão da barra crítica ou de uma barra específica, a perda total de potência ativa $(\mathrm{Pa})$, o coeficiente angular da reta e equações de parábolas.

As principais equações de cada método foram apresentadas visando um melhor entendimento e mostrando o que cada método tem em comum.

\section{AGRADECIMENTOS}

Os autores agradecem a UNESP e ao CNPq pelo apoio financeiro.

\section{REFERÊNCIAS}

[1] V. Ajjarapu and C.Christy. The Continuation Power Flow: a Tool for Steady State Voltage Stability Analysis. IEEE Trans. on Power Systems, vol. 7, n. 1, February, p. 416-423, 1992.

[2] A. Bonini Neto, D. A. Alves. Técnica de Parametrização Geométrica para o Fluxo de Carga Continuado Baseado nas Variáveis Tensão Nodal e Fator de Carregamento. Controle \& Automação (Impresso), v. 19, p. 350366,2008

[3] A. Bonini Neto, E. M. Magalhães, D. A. Alves. Dishonest Newton Method Applied in Continuation Power Flow Through a Geometric Parameterization Technique. Revista IEEE América Latina, v. 14, p. 161170, 2016.

[4] E Garbelini, D. A. Alves, A. Bonini Neto, E. Righeto, L. C. P. da Silva, C. A. Castro. An efficient geometric parameterization technique for the continuation power flow. Electric Power Systems Research, vol. 77, p. 71-82, 2007.

[5] A. Bonini Neto, D. A. Alves. Improved geometric parameterization techniques for continuation power flow. IET Generation, Transmission \& Distribution; vol. 4, p. 1349-1359, 2010.

[6] E. M. Magalhães, A. Bonini Neto, D. A. Alves. Técnica de Parametrização Geométrica para o Fluxo de Carga Continuado Baseado nas Variáveis Fator de Carregamento e Perda Total de Potência Ativa. In: Décimo Quinto Encontro Regional Ibero-Americano do Cigré - XV ERIAC, Foz do Iguaçu, p. 1-8, 2013.

[7] H. D. Chiang, H. Li, H. Yoshida, Y. Fukuyama, Y. Nakanishi. The generation of ZIP-V Curves for Tracing Power System Steady State Stationary Behavior Due to Load and Generation Variations. IEEE PES Summer Meeting, Edmonton, Alberta, Canada, p. 647-651, 1999.

[8] R. Seydel, "From Equilibrium to Chaos: Pratical Bifurcation and Stability Analisys", 2th. ed., Springer-Verlag: New York, 1994.

[9] H. D. Chiang, A. J. Flueck, K. S. Shah, and N. Balu. "CPFLOW: A practical tool for tracing power systems steady-state stationary behavior due to load and generation variations", IEEE Trans. Power Systems, vol. 10, p. 623-634, 1995.

[10] J. Zhao, B. Zhang. Reasons and countermeasures for computation failures of continuation power flow. In: PROCEEDINGS OF THE IEEE POWER ENGINEERING SOCIETY GENERAL MEETING (PES '06), June 2006.

[11] S. H. Li, H. D. Chiang. Nonlinear predictors and hybrid corrector for fast continuation power flow, IET Generation, Transmission \& Distribution; vol. 2, p. 341-354, 2008. 
[12] A. Bonini Neto, E. M. Magalhães, D. A. Alves. Obtaining the Maximum Loading Point of Electric Power Systems using the Decoupled Methods of Newton. Revista IEEE América Latina, v. 14, p. 371-379, 2016.

[13] E. M. Magalhães, A. Bonini Neto, D. A. Alves. A parameterization technique for the continuation power flow developed from the analysis of power flow curves. Mathematical Problems in Engineering, vol. 2012, p. 1-24, 2012.

[14] D. A. Alves, L. C. P. da Silva, C. A. Castro, V. F. da Costa. Study of Alternative Schemes for the Parameterization Step of the Continuation Power Flow Method Based on Physical Parameters-Part-I: Mathematical Modeling. Electric Power Components and Systems, Estados Unidos, v. 31, n.12, p. 1151-1166, 2003.

[15] D. A. Alves, L. C. P. da Silva, C. A. Castro, V. F. da Costa. Study of Alternative Schemes for the Parameterization Step of the Continuation Power Flow Method Based on Physical Parameters-Part-II: Performance Evaluation. Electric Power Components and Systems, Estados Unidos, v. 31, n.12, p. 1167-1177, 2003.

[16] MATHWORKS. Matlab (MATrix LABoratory). Disponível em: $<$ http://www.mathworks.com>. Acesso em 21 de maio de 2016.

[17] A. Bonini Neto, D. A. Alves, E. de M. Magalhães. Estudos de singularidades no fluxo de carga continuado. In: Romes Antonio Borges; Thiago Alves de Queiroz. (Org.). Matemática aplicada à indústria: problemas e métodos de solução. 1ed. São Paulo: Editora Blucher, p. 1742, 2016.

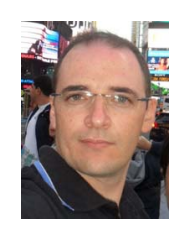

A. Bonini Neto, graduado em Matemática pela Faculdade de Dracena - UNIFADRA, Brasil, em 2002, mestrado e doutorado em Engenharia Elétrica pela Universidade Estadual Paulista - UNESP, campus de Ilha Solteira em 2006 e 2011 respectivamente. Trabalha com pesquisas na área de matemática aplicada na engenharia, técnicas de parametrização no fluxo de carga continuado. Atualmente é professor assistente doutor da Universidade Estadual Paulista - UNESP, campus de Tupã.

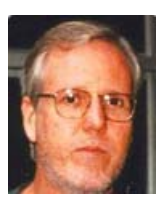

D. A. Alves, graduado em Engenharia Elétrica pela Universidade de São Paulo - USP, Brasil em 1980, mestrado e doutorado em Engenharia Elétrica pela Universidade Campinas - UNICAMP em 1988 e 2000 respectivamente. Trabalha com pesquisas na área de fluxo de carga continuado. Atualmente é professor adjunto da Universidade Estadual Paulista - UNESP, campus de Ilha Solteira. 